\title{
Linking glacial and future climates through an ensemble of GCM simulations
}

\author{
J. C. Hargreaves ${ }^{1}$, A. Abe-Ouchi ${ }^{1,2}$, and J. D. Annan ${ }^{1}$ \\ ${ }^{1}$ FRCGC/JAMSTEC, Yokohama, Japan \\ ${ }^{2}$ CCSR, Tokyo, Japan
}

\begin{abstract}
In this paper we explore the relationships between the modelled climate of the Last Glacial Maximum (LGM) and that for doubled atmospheric carbon dioxide compared to the pre-industrial climate by analysing the output from an ensemble of runs from the MIROC3.2 GCM.

Our results lend support to the idea in other recent work that the Antarctic is a useful place to look for historical data which can be used to validate models used for climate forecasting of future greenhouse gas induced climate changes, at local, regional and global scales. Good results may also be obtainable using tropical temperatures, particularly those over the ocean. While the greater area in the tropics makes them an attractive area for seeking data, polar amplification of temperature changes may mean that the Antarctic provides a clearer signal relative to the uncertainties in data and model results. Our result for Greenland is not so strong, possibly due to difficulties in accurately modelling the sea ice extent.

The MIROC3.2 model shows an asymmetry in climate sensitivity calculated by decreasing rather than increasing the greenhouse gases, with $80 \%$ of the ensemble having a weaker cooling than warming. This asymmetry, if confirmed by other studies would mean that direct estimates of climate sensitivity from the LGM are likely to be underestimated by the order of half a degree. Our suspicion is, however, that this result may be highly model dependent. Analysis of the parameters varied in the model suggest the asymmetrical response may be linked to the ice in the clouds, which is therefore indicated as an important area for future research.
\end{abstract}

\section{Introduction}

Paleoclimate simulations provide an opportunity to validate model performance under substantially different conditions to the modern climate. Nevertheless, most effort in climate modelling still goes towards improving the models' representation of the details of the present day climate. Recent

Correspondence to: J. C. Hargreaves

(jules@jamstec.go.jp) work (Annan et al., 2005) has, however, shown that this is by no means a guarantee of success: it is possible to improve the representation of the present day (quasi steady-state) climate in a model while simultaneously decreasing the accuracy of its representation of climate change in response to substantial historical changes in boundary conditions (and therefore presumably worsening predictions of future change). Therefore, it is important to consider whether there are other ways of gaining confidence in, and improving the accuracy of, model predictions.

The last glacial maximum (LGM) epoch has long been recognised as a time which might provide useful information for inferring future climate changes (eg Manabe and Brocolli, 1985), due to the fact that it is the most recent time (and therefore the time for which paleoclimate data is available in some quantity and quality) when forcings, (including those from greenhouse gases), and the climate state itself, were significantly different from the modern era. Since the net forcing at that time was strongly negative, and includes large contributions from factors other than greenhouse gas levels (most notably, large ice sheets in the northern hemisphere), it is unclear as to how directly we can infer future climate changes based on the LGM state. Nevertheless, there is still useful evidence here, especially when considered in combination with other lines of evidence such as the modern warming trend, and the short-term response to volcanic forcing, which are individually somewhat weak but collectively rather more convincing (Annan and Hargreaves, 2006). Furthermore, even if paleoclimate simulations provide only limited validation of climate predictions, not undertaking such studies at all could hardly be argued to be a better strategy.

Annan et al. (2005) found a correlation between modelled LGM (global and tropical) $2 \mathrm{~m}$ temperature (T2) change and global T2 change (compared to the modern climate) for doubled atmospheric carbon dioxide $\left(2 \times \mathrm{CO}_{2}\right)$ in the MIROC3.2 GCM (Hasumi and Emori, 2004). In that work the data used to validate the model's LGM state were the PMIP1 Alkenone data (http://www-lsce.cea.fr/pmip/, Harrison, 2000) from from the tropical ocean region. These data

Published by Copernicus GmbH on behalf of the European Geosciences Union. 
have been widely used (eg Houghton et al., 2001, Chapter 8) and provide coverage over a substantial proportion of the Earth's surface, so were therefore assumed to be reasonably representative of global climate change, but this question is still very much open. The availability and precision of regionally inhomogeneous data, the understanding of the forcings that dominate over particular geographical areas, and the confidence with which past and future changes can be linked are all factors which may affect which data are most useful for validating and improving model performance.

A recent examination of a multi-model ensemble from a range of different experiments (broadly PMIP1, PMIP2 and CMIP; http://www-lsce.cea.fr/pmip/, http://www-lsce. cea.fr/pmip2/ and http://www-pcmdi.llnl.gov/projects/cmip/ index.php respectively) was undertaken by Masson-Delmotte et al. (2006) (hereafter MD06), with the focus of assessing the potential value of polar ice cores for providing "quantitative insights on global climate change". Although their results were somewhat inhibited by small sample statistics, they concluded that there was a clear correlation between the global average and polar temperature changes compared to the control climates in the models for both the LGM and increased $\mathrm{CO}_{2}$ experiments. However, due to very limited overlap between the model populations which were integrated for both the LGM, and increased $\mathrm{CO}_{2}$ states, they did not in fact analyse whether the polar or global LGM temperature changes were related, in the models, to the global or polar temperature changes for the increased $\mathrm{CO}_{2}$ states, although they considered their results to be consistent with the hypothesis that such a relationship does exist. Crucifix (2006) investigated this question with the set of 4 models for which both LGM and doubled $\mathrm{CO}_{2}$ integrations are available, and found no evidence of a relationship between global or tropical temperature changes. With only 4 coupled atmosphere-ocean model runs available which covered a modest range of climate sensitivity, it is not yet clear to what extent LGM simulations can help to narrow the rather wider range of model results that has sometimes been presented as plausible (e.g. Andronova and Schlesinger, 2001; Stainforth et al., 2005). Schneider von Deimling et al. (2006) found a strong relationship between $\mathrm{LGM}$ and $2 \times \mathrm{CO}_{2}$ conditions across an ensemble of intermediate complexity climate model with uncertain parameters allowed to vary, but Annan et al. (2005) found a rather weaker relationship with a more sophisticated model (state of the art AGCM with slab ocean) which has more sources of uncertainty in atmospheric feedbacks.

In this paper we extend our previous analysis and consider further the conclusions presented by MD06, by examining a large perturbed-parameter ensemble from one particular model (MIROC3.2 Hasumi and Emori, 2004). While we are able to integrate numerous pairs of identical model versions for both LGM and $2 \times \mathrm{CO}_{2}$ climates and therefore do not have such a severe problem with small sample statistics, our results are necessarily tentative because we show results from only one model, and as MD06 showed, results can vary considerably between different models. In addition, for computational reasons we are using the model in a slab ocean configuration, rather than the fully coupled model which is now state-of-the-art for PMIP2. However, our results suggest areas where further investigations may be worthwhile with a wider range of models. Also, where even a single-model ensemble generates negative or weak results, it seems unlikely that a multi-model ensemble, which introduces more sources of uncertainty, will generate anything more useful.

We broaden the scope of the MD06 work, by considering not only annual average temperatures at the poles, but consider more broadly the zonal variation, the effects of land and ocean and also the seasonal variations. The main motivation for this is that data are available at a wide range of latitudes, and some are plausibly considered more directly representative of seasonal changes (e.g. precipitation-dependent proxies) rather than annual averages.

In order to further explore the value of the LGM climate for estimating climate sensitivity we also compare the results from an experiment where we do not impose massive ice sheets or the insolation forcing of the LGM state, and thus the only change compared to the control run is that the levels of greenhouse gases (GHG) are changed to the LGM levels prescribed by PMIP2.

In Sect. 2 we outline the way the ensemble of model runs was formed and discuss the climate states that were modelled. In Sect. 3 we discuss the results focusing principally on a zonal analysis of the T2 temperature changes. In Sect. 4 we discuss the implications of our results for the calculation of climate sensitivity. In Sect. 5 we briefly touch on the complex issue of the attributing climate changes to variation in individual parameters, and then we conclude with an overview of the results and discussion of the wider implications.

\section{Methods}

\subsection{Ensemble of MIROC3.2 runs}

For these experiments we use the T21L20 slab-ocean version of the state-of-the art GCM MIROC3.2 (Hasumi and Emori, 2004). The atmospheric component is a reduced-resolution version of the standard T42 version used in several modelling studies, including the results analysed by MD06 and Crucifix (2006). The physical and numerical schemes are unchanged, and a "control run" (with the parameter values taken directly from the control T42 model, with the exception of the strongly resolution-dependent gravity wave drag parameter) produced similar results to those of the higher resolution model at both LGM and $2 \times \mathrm{CO}_{2}$ states. We used the ensemble Kalman filter (EnKF) to generate three ensembles each of 40 members (Annan et al., 2005). For each experiment, we used the same expert opinion for the prior ranges of 25 parameters which we allowed to vary. The model was tuned to 
seasonally-averaged (summer and winter only) fields of 15 different climatological variables such as temperature, precipitation, radiation and winds. The only difference between the three experiments was in the judgment as to the model error that we considered reasonable. One ensemble consists of models which actually reproduce the climate fields better (as indicted by a normalised RMS error measure) than the control run, and the other two were less tightly tuned to the data and so covered a wider range of the parameter space. The experiment is described more fully in Annan et al. (2005). Creation of the original ensembles is time consuming and computationally expensive and the study presented in this paper uses the output from these previous experiments. For simplicity and in order to achieve improved statistics we combine the three ensembles and analyse them as one large ensemble. Taken as a whole we have a set of runs which all compare reasonably well with present day climatology but with different values for all the 25 varied parameters. A general understanding of model error (sometimes called "discrepancy": see Rougier (2006) for more discussion) is at present rather limited, and the model results exhibit a bias towards high sensitivity that we do not consider to be a realistic representation of our overall uncertainties (further investigations and development of the model is ongoing) so we simply combine the three ensembles in our analysis to explore the emergent relationships between different climate states that appear significant in the context of our experiment.

\subsection{Model runs}

After the parameter sets were generated, we then performed 4 experiments with all the model instances: pre-industrial (CTRL) climate, doubled $\mathrm{CO}_{2}\left(2 \times \mathrm{CO}_{2}\right), \mathrm{LGM}$ (with PMIP2 boundary conditions) and LGMGHG (greenhouse gases and orbital parameters as for PMIP2, but without the ice sheet and insolation changes of the PMIP2 protocol). Table 1 gives an overview of the forcings for the 4 experimental model climates. The experiments were run until the annual average temperatures had converged (at least 24 years for LGM and LGMGHG, 36 years for $2 \times \mathrm{CO}_{2}$ ) and then a further 20 years were averaged for the climatological results discussed below.

The 120 member ensemble was run for each of the $4 \mathrm{ex}-$ periments, but only 119 runs were used in the analysis. One model run, under LGMGHG boundary conditions, exhibited runaway cooling with no sign of equilibrating over a 50 year integration. Strong cooling was centred on the eastern equatorial Pacific. This behaviour appears to be due to the same phenomenon as that noted by Stainforth et al. (2005) (a nonphysical localised cooling instability arising from the limitations of a slab ocean model), and we therefore exclude this member from all of our analyses. Since we are seeking to analyse the relevance of paleo-temperature data for future temperature change prediction, we confine our analysis here to consideration of the modelled surface $(2 \mathrm{~m})$ temperatures. We have analysed the 119 member ensemble to look at the
Table 1. Overview of the forcings imposed for the 4 experimental climates. The forcings labelled "PMIP2" refer to the forcings for the PMIP2 $21 \mathrm{kyr}$ experiment, for which the ice sheet is ICE5G V1.1 (Peltier, 2004).

\begin{tabular}{|c|c|c|c|c|c|}
\hline \multirow[t]{2}{*}{ run } & \multicolumn{3}{|c|}{ GHG } & \multirow[t]{2}{*}{ insolation } & \multirow[t]{2}{*}{ ice sheet } \\
\hline & $\begin{array}{l}\mathrm{CO}_{2} \\
(\mathrm{ppm})\end{array}$ & $\begin{array}{l}\mathrm{N} 2 \mathrm{O} \\
(\mathrm{ppb})\end{array}$ & $\begin{array}{l}\mathrm{CH} 4 \\
(\mathrm{ppb})\end{array}$ & & \\
\hline CTRL & 285 & 280 & 860 & CMIP & CMIP \\
\hline $2 \times \mathrm{CO}_{2}$ & 570 & 280 & 860 & CMIP & CMIP \\
\hline LGM & 185 & 200 & 350 & PMIP2 & PMIP2 \\
\hline LGMGHG & 185 & 200 & 350 & CMIP & CMIP \\
\hline
\end{tabular}

correlations between several different components of both model variables, and also the relationship of these with the parameters. The correlations indicate the extent to which our uncertainties about the climate system (as encapsulated by imperfectly known parameter values in the model equations) affect past and future climate simulations in similar ways. Where the historical simulation is weakly related to the future, then increasing our skill in this aspect of the simulation will hardly affect our predictions, even if it does increase our understanding of some physical processes. Conversely, a strong relationship would suggest that simulations which were quantitatively improved in this area could reasonably be expected to give a more accurate and reliable forecast.

For 119 independent samples from a distribution, the 99\% significant correlation coefficient from the student $\mathrm{T}$ test, assuming a independent samples from a normal distribution, is 0.24 . Our ensemble is a somewhat ad-hoc mixture of three 40 member ensembles, so, in the rather qualitative discussion in this paper, we use this value as a guide as to the strength of the correlation rather than a definitive threshold. It is also possible that a different experiment with MIROC3.2, varying different parameters and making different prior assumptions could produce an ensemble of equally reasonable model runs with rather different resultant characteristics. Due to the substantial investment in time required to perform this experiment (several months), we have not yet undertaken a repeat experiment of this nature, although one is planned for the future which will also use a revised and updated version of the model. In the following discussion we consider a correlation above 0.5 to be strong and one below 0.3 to be weak. Since our model has a relatively low-resolution T21 grid, we do not expect accurate results at the grid-point level for comparison with in-situ data. Therefore, we focus on zonal averages rather than the location-based estimates. However, for comparison with the MD06 results we have also derived some results for Greenland and Antarctica. It should also be noted that it was recently discovered that this version of the model contained a bug which generated a bias in the air temperatures over land ice. The bias induced in zonal $\mathrm{T} 2$ changes at 

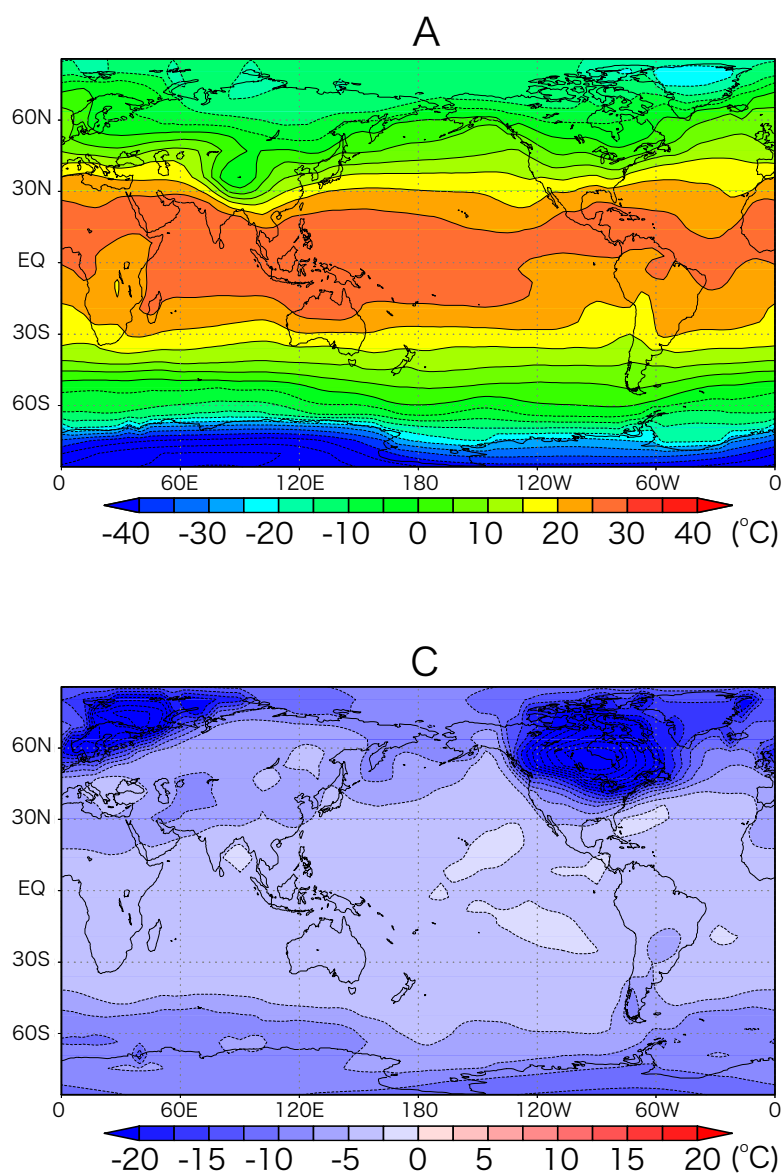
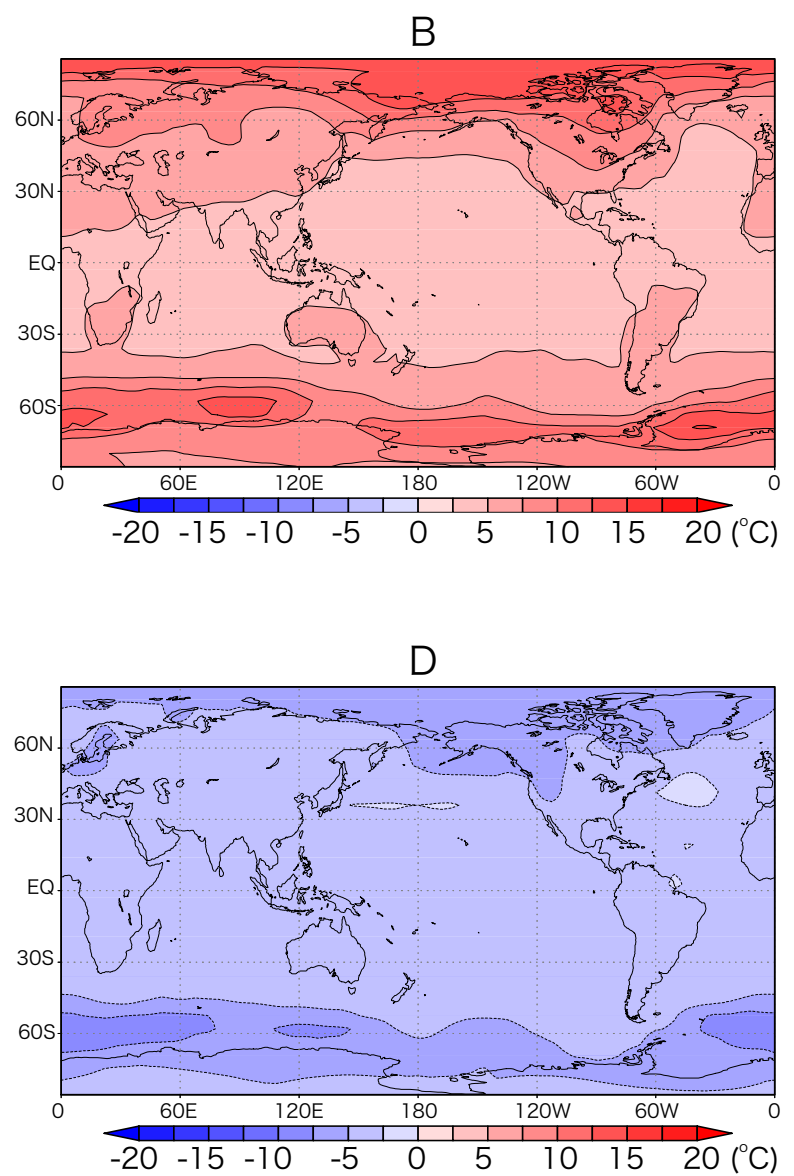

Fig. 1. Annually averaged T2 temperature: mean of the 119 MIROC3.2 ensemble members. A: Control (CTRL) run, B: (2xCO $2-C T R L)$, C: (LGM-CTRL), D: (LGMGHG-CTRL).

the LGM in the T42 model is close to $1^{\circ} \mathrm{C}$ north of $80^{\circ} \mathrm{N}$ and much smaller than that south of this latitude. As shown in Fig. 2 this is a very small fraction of the temperature changes at the LGM. Although due to computational limitations we have not been able to test it, the bias is small enough that we do not expect it to vary greatly across our ensemble. We therefore think it unlikely that this will have significantly affected our analysis which focuses not on the magnitude of temperature changes themselves but on the correlations between the temperature changes for the three experimental climate states.

\section{Correlation between LGM and doubled $\mathrm{CO}_{2}$ temper- ature changes}

The ensemble mean, annually averaged $\mathrm{T} 2$ results are shown in Fig. 1, with sub-plot A showing the CTRL results and the other three sub-plots showing the differences in temperature between the each specific climate state and the CTRL. The zonal temperature changes for December, January and
February (DJF) and June, July and August (JJA) for the three experiments and also the actual average temperatures for the control run are illustrated in Fig. 2. The existence of a strong "polar amplification" (as discussed by MD06) of the temperature changes can be seen in the results from this model.

Crucifix (2006) quotes the following observational estimates of climate change: Antarctica, $-9 \pm 2{ }^{\circ} \mathrm{C}$ (Jouzel et al., 2003); Greenland, $-20 \pm 2{ }^{\circ} \mathrm{C}$ (Cuffey and Clow, 1997; DahlJensen et al., 1998); and the tropical ocean, $-2.7 \pm 0.5^{\circ} \mathrm{C}$ (Ballantyne et al., 2005; Lea, 2005). For comparison with these results we have the following mean and 1 standard deviation range for our ensemble: Antarctica, $-9 \pm 1.3^{\circ} \mathrm{C}$; Greenland, $-18 \pm 2{ }^{\circ} \mathrm{C}$; tropical ocean, $-3.0 \pm 0.5^{\circ} \mathrm{C}$. Here we quote the average $2 \mathrm{~m}$ temperatures over the Greenland and Antarctic land masses and the tropical region includes the ocean grid boxes between latitudes $30^{\circ} \mathrm{S}$ and $30^{\circ} \mathrm{N}$. While interactive vegetation models are included in the PMIP2 protocol, the PMIP2 boundary conditions for AGCMs and AOGCMs exclude the effects of some vegetation and dust forcings which are thought to be significant and negative. This supports our belief that our ensemble of models has an 

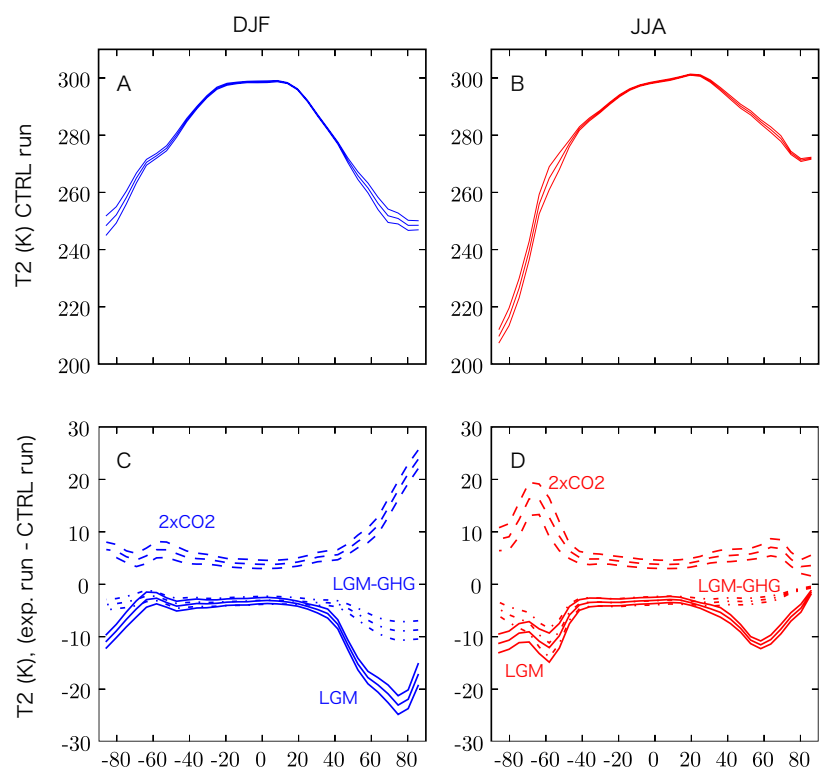

Latitude $\left({ }^{\circ} \mathrm{N}\right)$

Fig. 2. Top plots: Seasonally averaged (left DJF; right JJA) zonal T2 profiles for the control (CTRL) climate. Lower plots: Dashed line, $\left(2 \times \mathrm{CO}_{2}\right.$-CTRL); solid line, (LGM-CTRL); dot-dashed line, (LGMGHG-CTRL). The lines show the mean and one standard deviations of the 119 member ensemble results.

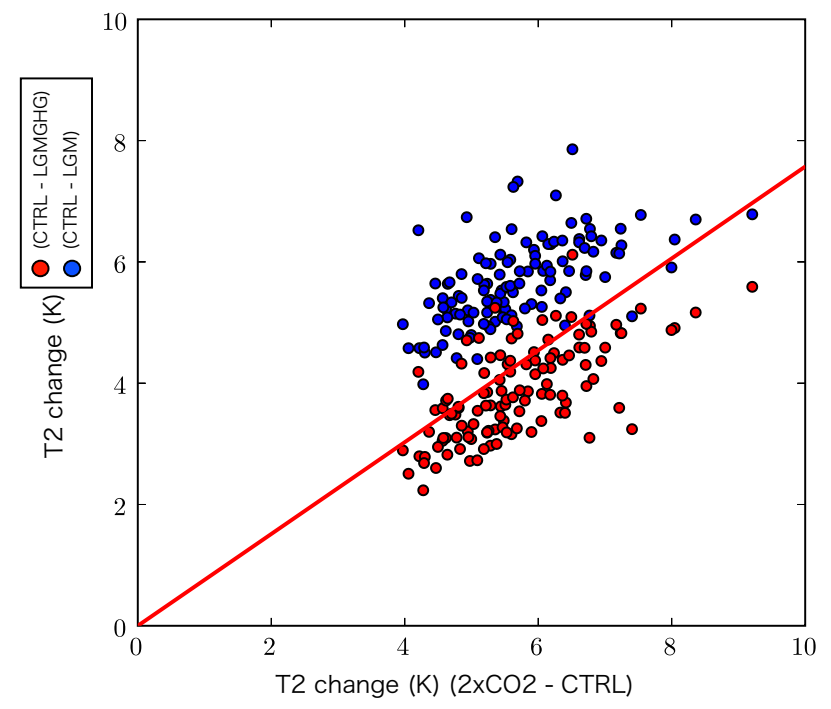

Fig. 3. Scatter plot of the magnitude of global annually averaged temperature change (all changes are magnitudes relative to CTRL state) for $2 \times \mathrm{CO}_{2}$ vs. LGM (blue), $2 \times \mathrm{CO}_{2}$ vs LGMGHG (red), for the 119 member model ensemble. The red line shows the expected results for the LGMGHG and $\mathrm{CO}_{2}$ experiments if the response to increased and decreased GHG concentrations were linear, in forcing.
Table 2. Correlation coefficients for the magnitude of tropical and global T2 temperature changes with respect to the CTRL state for the three climates. Only the correlations mentioned in the text are shown. "gl." denotes globally averaged T2 changes, and "tr." denotes averages from the tropical region $(30 \mathrm{~S}-30 \mathrm{~N})$

\begin{tabular}{lccccc}
\hline & $2 \times \mathrm{CO}_{2}$ & \multicolumn{2}{c}{ LGM } & \multicolumn{2}{c}{ LGMGHG } \\
& tr. & gl. & tr. & gl. & tr. \\
\hline $2 \times \mathrm{CO}_{2}$ gl. & 0.94 & -0.59 & -0.65 & -0.67 & -0.64 \\
$2 \times \mathrm{CO}_{2}$ tr. & & & -0.69 & & \\
LGM gl. & & & 0.95 & 0.92 & 0.81 \\
\hline
\end{tabular}

overall bias towards high sensitivity. That is, with a more complete set of forcings, our models would have shown a clearly stronger cooling than that observed.

\subsection{Global and tropical analysis}

Figure 3 shows scatter plots for the globally averaged T2 changes for both LGM and LGMGHG verses $2 \times \mathrm{CO}_{2}$, illustrating the smaller $\mathrm{T} 2$ changes (as expected) for LGMGHG. The correlation between the $\mathrm{T} 2$ changes is clear. The correlation coefficients for these results and some others are given in Table 2. The correlation coefficient is stronger between $2 \times \mathrm{CO}_{2}$ and LGM climates when looking at the tropics only. The LGMGHG global T2 change is more highly correlated with LGM than $2 \times \mathrm{CO}_{2}$ climates. This is perhaps surprising since, if the response to changes in greenhouse gas forcing was linear across the range covered by the $2 \times \mathrm{CO}_{2}$ and LGMGHG states, then one would expect the correlation between these two states to be the stronger, because the LGM climate state is also strongly influenced by the large ice sheet and to a lesser extent by changes in solar forcing. It seems, therefore, that a large proportion of uncertainty in the model response is due to a nonlinearity in the response to positive and negative forcings, which we discuss further in Sect. 4.

\subsection{Zonal analysis}

Here we consider how the globally and zonally averaged patterns of temperature change are correlated for the different experiments.

\subsubsection{Doubled $\mathrm{CO}_{2}$ experiment}

The dashed line in Subplot A of Fig. 4 shows that, unsurprisingly, the correlation between the temperature changes for global and zonally averaged temperature change for the $2 \times \mathrm{CO}_{2}$ climate is strong at all latitudes, although there is a notable drop in the southern sea-ice region (around $65^{\circ} \mathrm{S}$ ). Small changes in sea ice extent cause large localised temperature changes due to the positive feedback of the albedo 


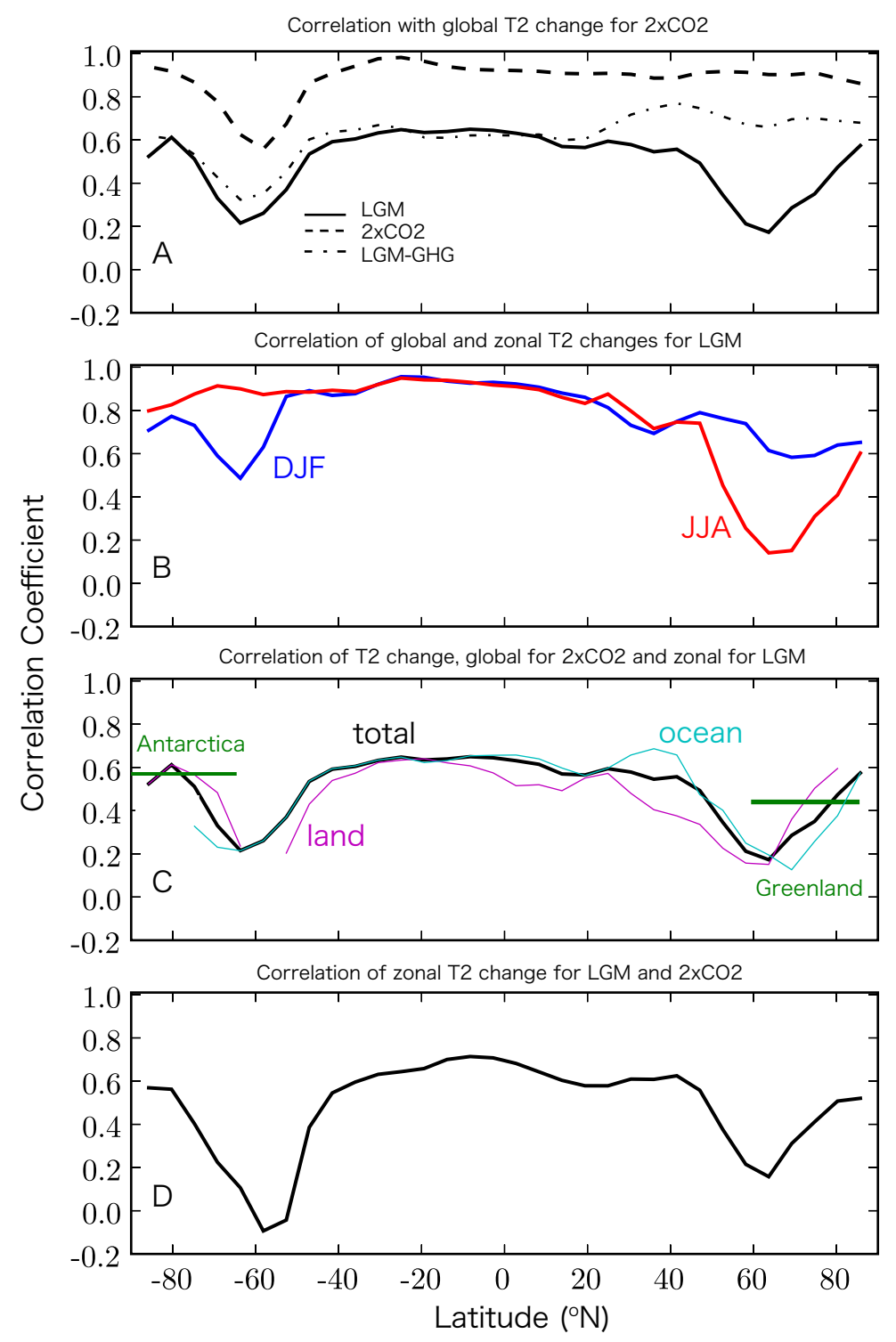

Fig. 4. Correlations between some global and zonally-averaged temperature changes (all changes relative to CTRL state): A: Globally averaged T2 for $2 \times \mathrm{CO}_{2}$ and zonal averages for: $2 \times \mathrm{CO}_{2}$ (dashed); LGM (solid); LGMGHG (dot-dashed). B: Annual Globally and seasonally zonally averaged T2 changes for LGM: DJF (blue); JJA (red). C: Globally averaged T2 change for $2 \times \mathrm{CO}_{2}$ and zonally averaged LGM: black (land+ocean), cyan (ocean only), magenta (land only). D: Zonally averaged T2 change for LGM and $2 \times \mathrm{CO}_{2}$.

effect. Even with ocean heat fluxes calculated to reasonably reproduce the present day climate, the ensemble members have somewhat different sea-ice extents in the modern climate, which results in substantially different temperature changes in this region when the ice extent shrinks (vanishes) in the warmer climate. Thus, the temperature change is strongly influenced by small biases in the initial sea ice extent.

\subsubsection{LGM experiment}

There is also also generally a high correlation between the global and zonally averaged temperature change at the LGM. Figure 4 subplot B shows this result split into DJF and JJA seasons. Both polar sea-ice regions (but not the poles themselves) show markedly lower correlation in the summer seasons, falling away to nothing during JJA for northern high latitudes, where the northern hemisphere ice sheets and sea ice are located.

With a lack of identical models run for both $2 \times \mathrm{CO}_{2}$ and 
LGM conditions, the relationship between the two was assumed by MD06. Here we examine the relationship between the LGM and climate sensitivity by looking at the correlation between the magnitudes of the zonally averaged LGM and globally averaged $2 \times \mathrm{CO}_{2}$ temperature changes. This result is shown as the solid black lines in subplots $\mathrm{A}$ and $\mathrm{C}$ of Fig. 4. While still high in places (including Antarctica), it is considerably lower (especially at northern latitudes) than the correlation between global and zonally averaged LGM temperature change. Areas of strong correlation include both central Antarctica and the tropics. The other two lines in subplot $\mathrm{C}$ of Fig. 4 shows the annually averaged results for the same correlation, split into land (magenta) and ocean (cyan). This shows a generally better correlation with the temperature over the ocean than the land between the latitudes of $50^{\circ} \mathrm{S}$ and $50^{\circ} \mathrm{N}$. Also shown are the values of the correlation coefficients for the averages over the Antarctica and Greenland land areas. These show that while the MD06 conclusions are supported with a high correlation for Antarctica, in MIROC3.2 there is not such a high correlation for Greenland. The MD06 results were for central Greenland $(>1300 \mathrm{~m})$ and central Antarctica $(>2500 \mathrm{~m})$. Although there are differences $\left(<2^{\circ} \mathrm{C}\right)$ in the magnitude of the temperature change, there is not a significant difference in the correlation coefficients evaluated using the central values rather than the averages. Due to the coarse resolution of our model we show the average land mass values since these are more likely to be robust.

Our results suggest that the tropics, particularly the ocean regions, may also be good places for calibrating and improving models which are then to be used for prediction of future climate change caused by increased greenhouse gas levels. The existence of this particular correlation in the same model has already been used in previous work (Annan et al., 2005), where we attempted to constrain estimates of climate sensitivity using tropical SST data from the LGM. Our results here show that including Antarctic temperature estimates from ice cores into the calculation could potentially improve the result from such an experiment. Despite the small area at the poles, the data there may be less noisy than at the tropics due to the fact that the total temperature changes (Figs. 1 and 2) are much greater for the polar regions than the tropics in the winter months (and for the annual mean) for both $2 \times \mathrm{CO}_{2}$ and the LGM. Similar conclusions have also been drawn by Schneider von Deimling et al. (2006). Correlations in the sea ice regions and over zones where the Northern ice sheets are situated at the LGM are weak, suggesting that, at least in our model, these regions are less informative of future climate changes.

Understanding and predicting climate change at smaller scales than global is obviously desirable. In this context we would like to know to what extent LGM climate changes can be used to validate the predictive models at the regional scale. As a step towards this we have calculated the correlation between the magnitude of the zonally averaged temperature changes for LGM and $2 \times \mathrm{CO}_{2}$ climates. The resulting vari- ation of the correlation coefficient with latitude is similar in shape to that obtained from analysing the globally averaged $2 \times \mathrm{CO}_{2}$ and zonally averaged LGM changes. The correlation in the tropical regions is stronger, while insignificant in the southern sea-ice region. This strengthening in those areas that were strongly correlated with global changes might be expected, while the weakening in the sea-ice region indicates that, further to the discussion in Sect. 3.2.1, the large non-linear albedo feedback is such that the small differences in the modelled extent of sea ice leads to large differences in the local temperature response to forcing changes.

\section{Implications for climate sensitivity}

Correlation coefficients for the LGMGHG experiment, where only the greenhouse gases were changed to LGM levels but all other forcings were kept the same as the control run, are shown in Table 2. As already discussed in Sect. 3.1 the LGMGHG temperature changes are more strongly correlated with the LGM temperature changes than the $2 \mathrm{xCO}_{2}$ temperature changes. As is apparent from Fig. 3 there is almost as much scatter in the LGMGHG vs $2 \times \mathrm{CO}_{2}$ (red) temperatures as there is for the LGM vs $2 \times \mathrm{CO}_{2}$ temperatures (blue). This is a somewhat surprising result which implies that the uncertainty in the response to the ice sheet does not outweigh that due only to the nonlinearity in the response to increasing versus decreasing GHG levels. Looking at the dot-dashed line in Subplot A of Fig. 4 which shows the correlation between the magnitudes of the global temperature change for $2 \times \mathrm{CO}_{2}$ and zonal temperature change for LGMGHG, the line more closely follows the LGM zonal variation except north of about $30^{\circ} \mathrm{N}$ where the correlation is more like the $2 \times \mathrm{CO}_{2}$ zonal variation. So, while the north of the northern hemisphere is largely influenced by the ice sheets at the LGM, it seems that uncertainty in the influence of the ice sheet does not have a clear influence on the rest of the globe and therefore it must be nonlinearity of the response to differing GHG levels across the range tested that produces a large part of the observed scatter in the relationship between LGM and $2 \times \mathrm{CO}_{2}$ climates. The standard estimate for the radiative forcing due to changes in greenhouse gas levels at the LGM is approximately $-2.8 \mathrm{Wm}^{-2}$, whereas that due to doubling $\mathrm{CO}_{2}$ is $+3.7 \mathrm{Wm}^{-2}$ (Houghton et al., 2001 ), a ratio of -0.76 . The radiative forcing has been calculated in two version of the MIROC model (at T42 resolution, with different atmospheric parameterisations) and found to be in close agreement with this ratio, even though the absolute values were marginally different in each case (Yokohata et al., 2005). Since we did not alter any parameters directly relating to radiative transfer in our experiments, we expect this relationship to hold across our ensemble and although we have not performed a precise calculation, there is no sign of a correlation between the initial radiative imbalance (when GHG levels are changed abruptly) and the 
Table 3. Parameter definitions for 9 parameters which showed some evidence of significant correlation with T2 changes at global or tropical scales.

\begin{tabular}{ll}
\hline parameter & description \\
\hline prctau & e-folding time for ice precipitation $(\mathrm{m} 3 / \mathrm{kg} / \mathrm{s})$ \\
elamin & minimum entrainment factor for cumulus convection $(1 / \mathrm{m})$ \\
tefold & e-folding time for horizontal diffusion $($ day) \\
rhmcrt & Critical relative humidity for cumulus convection $(-)$ \\
vice0 & ice fall speed factor $(\mathrm{m} / \mathrm{s})$ \\
dffmin & minimim vertical diffusion coefficient $(\mathrm{m} 2 / \mathrm{s})$ \\
alp & gravity wave drag factor $(\mathrm{rad} / \mathrm{m})$ \\
snrfrs & snow amount required for refreshing snow albedo $(\mathrm{kg} / \mathrm{m} 2)$ \\
ray0 & Rayleigh friction e-folding time (day)
\end{tabular}

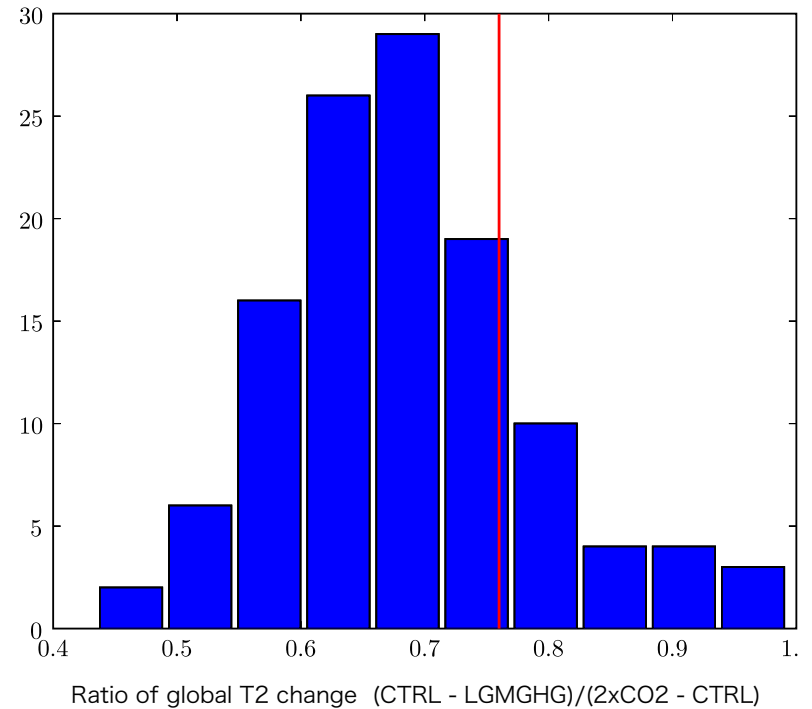

Fig. 5. Histogram of the ensemble results for the ratio of global T2 change for LGMGHG and $2 \times \mathrm{CO}_{2}$ experiments, T2(LGMGHG $\left.-\mathrm{CTRL}) / \mathrm{T} 2\left(2 \times \mathrm{CO}_{2}-\mathrm{CTRL}\right)\right)$. The red line at 0.76 indicates the point where, assuming current estimates of the LGM forcing from greenhouse gases, the cooling and warming caused by decreasing and increasing $\mathrm{CO}_{2}$ would be symmetrical. Close to $80 \%$ of the ensemble show a greater sensitivity to warming than cooling.

equilibrium temperature change. If the models exhibited a constant sensitivity across this range, the magnitude of the LGMGHG global temperature changes would be $76 \%$ of that for $2 \times \mathrm{CO}_{2}$. In Fig. 5 we show the histogram of the global temperature changes plotted as a ratio T2(CTRLLGMGHG)/T2(2xCO 2 -CTRL). Also shown, with a red line, is the 0.76 value corresponding to equal sensitivity. The line on Fig. 3 also shows the expected results for the LGMGHG and $\mathrm{CO}_{2}$ experiments if the response to increased and decreased GHG concentrations were linear.

For the median of the ensemble, the difference between the value of climate sensitivity and T2(CTRL-LGMGHG)/0.76 is $0.62^{\circ} \mathrm{C}$. Furthermore, close to $80 \%$ of the ensemble have a smaller magnitude of temperature change when greenhouse gases are decreased rather than increased. This result is rather similar to that obtained by Hansen et al. (2005), but other models may differ and this conclusion will remain rather tentative until others undertake similar investigations.

\section{Correlations between parameter values and tempera- ture changes}

An in depth discussion of the physical effects of all of the 25 parameters which varied in the EnKF experiments is beyond the scope of this paper (and perhaps of little interest to those using different models). Here we briefly describe the statistical behaviour of the most significant parameters along with their characteristics to the extent that they illustrate some the results described above.

Table 4 shows the correlations of the temperature differences between the experimental climate states and the control climate for 9 of the 25 parameters which were allowed to vary independently in the EnKF experiments. These 9 parameters (defined in Table 3 ) are the only ones which individually showed even a marginally (at the previously mentioned $1 \%$ level) significant correlation for any of the three experimental climates (considering T2 changes with respect to the CTRL climate) at the global or tropical scale.

At the global and tropical scale for the $2 \times \mathrm{CO}_{2}$ climate, the clearly dominant parameter is "prctau", but interestingly, this parameter is less dominant for the LGM and LGMGHG climate states. This parameter is the time scale for ice precipitation in the clouds. Therefore a higher value should correspond to a higher total amount of ice in the clouds. The relationship of ice, clouds and sensitivity is complex, but Tsushima et al. (2006) find less cloud ice to be linked to in a larger pole-ward shift in cloud water and therefore a reduced cloud albedo effect, amplifying the overall warming. However, at least for the two versions of MIROC considered 
Table 4. Columns 2-7: correlations of parameters with global and tropical annual average T2 change for three climate compared to present day. $1 \%$ significance for correlations with 119 samples is 0.24 . Those correlations greater than this value are marked in bold. See Table 3 for parameter definitions. Columns 8-9: as for columns 2-7 but showing the correlations with the parameters of the ratios of the T2 changes for the cooler and warmer climates.

\begin{tabular}{lcccccccc}
\hline \multirow{2}{*}{ parameter } & \multicolumn{2}{c}{$2 \mathrm{xCO}_{2}$} & \multicolumn{2}{c}{ LGM } & \multicolumn{2}{c}{ LGMGHG } & LGM/2xCO & LGMGHG/2xCO \\
& Global & Trop. & Global & Trop. & Global & Trop. & & \\
\hline prctau & $\mathbf{0 . 5 3}$ & $\mathbf{0 . 5 0}$ & -0.22 & $\mathbf{- 0 . 3 1}$ & $\mathbf{- 0 . 3 3}$ & $\mathbf{- 0 . 3 5}$ & $\mathbf{0 . 4 3}$ & 0.14 \\
elamin & -0.21 & $\mathbf{- 0 . 4 0}$ & $\mathbf{0 . 3 1}$ & $\mathbf{0 . 3 9}$ & $\mathbf{0 . 3 5}$ & $\mathbf{0 . 4 0}$ & -0.02 & 0.21 \\
tefold & 0.19 & $\mathbf{0 . 3 0}$ & $\mathbf{- 0 . 2 7}$ & $\mathbf{- 0 . 2 9}$ & $\mathbf{- 0 . 2 9}$ & -0.23 & 0.06 & -0.13 \\
rhmcrt & 0.20 & $\mathbf{0 . 2 4}$ & -0.17 & $\mathbf{- 0 . 3 0}$ & $\mathbf{- 0 . 2 6}$ & $\mathbf{- 0 . 2 9}$ & 0.08 & -0.11 \\
vice0 & -0.17 & -0.22 & $\mathbf{0 . 3 8}$ & $\mathbf{0 . 3 2}$ & $\mathbf{0 . 3 9}$ & $\mathbf{0 . 3 0}$ & 0.15 & $\mathbf{0 . 3 5}$ \\
dffmin & 0.07 & -0.06 & $\mathbf{0 . 3 0}$ & $\mathbf{0 . 3 0}$ & $\mathbf{0 . 3 9}$ & $\mathbf{0 . 4 0}$ & $\mathbf{0 . 3 6}$ & $\mathbf{0 . 5 9}$ \\
alp & -0.21 & -0.13 & $\mathbf{0 . 3 3}$ & $\mathbf{0 . 3 3}$ & 0.18 & 0.13 & 0.03 & 0.01 \\
snrfrs & -0.08 & -0.11 & $\mathbf{0 . 3 1}$ & 0.20 & 0.19 & 0.11 & 0.14 & 0.16 \\
ray0 & 0.19 & 0.13 & -0.20 & -0.23 & -0.22 & $\mathbf{- 0 . 2 5}$ & 0.03 & -0.09 \\
\hline
\end{tabular}

in that work (among other GCMs) and an intermediate unpublished version, their overall sensitivity to LGM boundary conditions is rather similar, indicating that this change in model formulation has little effect under strong cooling conditions.

The other 3 parameters which are significant for the $2 \times \mathrm{CO}_{2}$ temperature changes in the tropics are not very significant on the global scale. Zonal analysis (not shown) shows that this is caused by a sharp decrease in the correlation (or even opposite correlation in some cases) in the southern sea ice region. While the LGM temperature changes also show significant correlation with these parameters particularly in the tropics, the LGM picture is further complicated by effects from four other parameters. This result is consistent with the results in Table 2 and Fig. 3, which shows considerable scatter in the relationship between LGM and $2 \times \mathrm{CO}_{2}$ temperature changes. Of these 4 additional parameters, 2 (alp, snfrs) are not significant for LGMGHG. It is perhaps unsurprising that alp (gravity wave drag) and snfrs (related to albedo) are more strongly related to the temperature changes over the ice sheet. The overall similarity in the parameters that are significant for both LGMGHG and LGM climate changes is consistent with the general similarity of the results for these two climates shown in Sub-plot A of Fig. 4.

Columns 8 and 9 of Table 4 show the correlations of the ratios of the $\mathrm{T} 2$ temperature changes for $\mathrm{LGM} / 2 \mathrm{CO}_{2}$ and LGMGHG/ $2 \mathrm{CO}_{2}$ respectively. These ratios should indicate which parameters are linked to the scatter around the red line in Fig. 3 and the spread of the histogram in Fig. 5. "prctau" is important for the $\mathrm{LGM} / 2 \mathrm{CO}_{2}$ ratio, but "vice0" is more important for the LGMGHG/ $2 \mathrm{CO}_{2}$ ratio. "vice0" is a scaling factor on the ice fall speed so has a similar effect to "prctau" in that its value affects the total amount of ice in the clouds. It seems plausible that some of the asymmetrical effect between warming and cooling is, therefore, linked in this model to the distribution of ice in clouds. The other parameter important in the temperature ratios in columns 8 and 9 of Table 4 is "dffmin", the minimum vertical diffusion coefficient. Higher values of this parameter are expected to lead to warmer winter conditions at high altitudes, so the result here, which indicates that the parameter is more important for cooler than warmer climates, is reasonable.

\section{Conclusions}

The model results presented in this paper show that in the MIROC3.2 model there is a reasonably strong link between global and tropical temperature changes at the LGM and those for $2 \times \mathrm{CO}_{2}$. However, there is a considerable amount of noise in the correlation, even though we are only considering the results from one model. It is clear that different processes (controlled by different parameters) affect the response to strong positive and negative forcings, even when this forcing is limited to radiative forcing of greenhouse gases. The albedo and topographical influences of large ice sheets complicate matters further, at least at the local level. Unsurprisingly, the links between regional and global scales within the same experimental epoch are much stronger. With a lack of model runs from both climate states, MD06 assumed the link existed, while Crucifix (2006) obtained results from only 4 simultaneous models and perceived no such link at the global scale. While a perturbed-parameter ensemble can form a step towards increasing our understanding, it is unlikely to cover the full range of results that structurally different models can achieve. It is therefore important, if this link is to be better understood, that directly comparable integrations of both LGM and $2 \times \mathrm{CO}_{2}$ climates are performed for a larger number of GCMs in future. Furthermore, it would be helpful to ensure that the LGM boundary conditions actually represent reality as faithfully as possible, rather 
than representing a sensitivity analysis in which some potentially important (albeit poorly understood) elements are omitted. This is especially important if a direct comparison with data is to be attempted. Dust and vegetation changes may alter the pattern of response, and changes in ocean heat transport would increase the uncertainty of the results.

Our results lend support to the idea in MD06 that the LGM Antarctic is a good place to look for a data which can be used to validate models used for climate forecasting of future GHG induced climate changes, at local, regional and global scales. Good results may in principle be obtainable using tropical temperatures, particularly those over the ocean. While the greater area in the tropics makes them an attractive area for seeking data, polar amplification of temperature changes (apparent in Fig. 2) may mean that the Antarctic provides a clearer signal relative to the uncertainties in data and model results. Our result for Greenland is not so strong, possibly due to difficulties in accurately modelling the sea ice extent.

The areas occupied by the massive northern hemisphere ice sheets and sea ice at the LGM would appear to be very poor places to seek data of relevance to GHG forcing. Our results indicate that the temperature changes in those regions are controlled by different parameters for both LGM and for the southern sea ice region for the $2 \times \mathrm{CO}_{2}$ climate. This implies different processes at work in those regions which therefore means that changes observed at the present day in the southern sea ice locations would provide only relatively weak information on the value of future globally averaged warming.

The MIROC3.2 model shows an asymmetry in climate sensitivity calculated by decreasing rather than increasing the greenhouse gases, with $80 \%$ of the ensemble having a weaker cooling than warming. This asymmetry, if confirmed by other studies, would mean that direct estimates of climate sensitivity from the LGM are likely to be underestimated by the order of half a degree. However, this result may be model dependent. Analysis of the parameters varied in the model suggest the asymmetrical response may be linked to the behaviour of ice in the clouds, which is therefore indicated as an important area for future research.

Acknowledgements. We thank the K-1 Japan project members for support and discussion, and in particular Tokuta Yokohata for the radiative forcing calculations. This work was partially supported by the Research Revolution 2002 (RR2002) of the Ministry of Education, Sports, Culture, Science and Technology of Japan. The model calculations were made on the Earth Simulator of JAMSTEC. The research was partially supported by JSPS Grant-in-Aid for Scientific Research (B) no. 16340136.

Edited by: P. Braconnot

\section{References}

Andronova, N. G. and Schlesinger, M. E.: Objective estimation of the probability density function for climate sensitivity, J. Geophys. Res., 108, 22 605-22 611, 2001.

Annan, J. D. and Hargreaves, J. C.: Using multiple observationallybased constraints to estimate climate sensitivity, Geophys. Res. Lett., 33, L06704, doi:10.1029/2005GL025259, 2006.

Annan, J. D., Hargreaves, J. C., Ohgaito, R., Abe-Ouchi, A., and Emori, S.: Efficiently constraining climate sensitivity with paleoclimate simulations, SOLA, 1, 181-184, 2005.

Ballantyne, A. P., Lavine, M., Crowley, T. J., Liu, J., and Baker, P. B.: Meta-analysis of tropical surface temperatures during the Last Glacial Maximum, Geophys. Res. Lett., 32, L05712, doi:10.1029/2004GL021217, 2005.

Crucifix, M.: Does the Last Glacial Maximum constrain climate sensitivity, Geophys. Res. Lett., 33, L18701, doi:10.1029/2006GL027137, 2006.

Cuffey, K. M. and Clow, G. D.: Temperature, accumulation, and elevation in central Greenland through the last deglacial transition, Geophys. Res. Lett., 102, 26383-26396, 1997.

Dahl-Jensen, D., Modegaard, Gundestrup, N., Clow, G. D., Johnsen, S. J., Hansen, W., and Balling, N.: Past temperatures directly from the Greenland ice sheet, Science, 282, 268-271, 1998.

Hansen, J., Sato, M., Ruedy, R., Nazarenko, L., Lacis, A., Schmidt, G., Russell, G., Aleinov, I., Bauer, M., Bauer, S., Bell, N., Cairns, B., Canuto, V., Chandler, M., Cheng, Y., Genio, A. D., Faluvegi, G., Fleming, E., Friend, A., Hall, T., Jackman, C., Kelley, M., Kiang, N., Koch, D., Lean, J., Lerner, J., Lo, K., Menon, S., Miller, R., Minnis, P., Novakov, T., Oinas, V., Perlwitz, J., Perlwitz, J., Rind, D., Romanou, A., Shindell, D., Stone, P., Sun, S., Tausnev, N., Thresher, D., Wielicki, B., Wong, T., Yao, M., and Zhang, S.: Efficacy of climate forcings, J. Geophys. Res., 110, 268-271, doi:10.1029/96JD03436, 2005.

Harrison, S. P.: Palaeoenvironmental data sets and model evaluation in PMIP, in: Proceedings of the third PMIP workshop. WCRP111; WMO/TD-No. 1007, edited by: Braconnot, P., 9-25, La Huardire, Canada, 2000.

Hasumi, H. and Emori, S.: K-1 coupled model (MIROC) description, K-1 technical report 1, Tech. rep., Center for Climate System Research, University of Tokyo, 2004.

Houghton, J. T., Ding, Y., Griggs, D. J., Noguer, M., Linden, P. J. V. D., Dai, X., Maskell, K., and Johnson, C. A.: Climate Change 2001: Contribution of Working Group I to the Third Assessment Report of the Intergovernmental Panel on Climate Change, Cambridge University Press, 2001.

Jouzel, J., Vimeux, F., Caillon, N., Delaygue, G., Hoffman, G., Masson-Delmotte, V., and Parrenin, F.: Magnitude of isotope/temperature scaling for interpretation of central Antarctic ice cores, J. Geophys. Res., 108(D120), 4361, doi:10.1029/2002JD002677, 2003.

Lea, D. W.: The 100,000-yr cycle in tropical SST, greenhouse forcing, and climate sensitivity, J. Climate, 17, 2170-2179, 2005.

Manabe, S. and Brocolli, A. J.: A comparison of climate model sensitivity with data from the last glacial maximum, J. Atmos. Sci., 42, 2643-2651, 1985.

Masson-Delmotte, V., Kageyama, M., Charbit, P. B. S., Krinner, G., Ritz, C., Jouzel, E. G. J., Abe-Ouchi, A., Gladstone, M. C R. M., Hewitt, C. D., LeGrande, A. K. A. N., Marti, O., Merkel, 
U., Ohgaito, T. M. R., Otto-Bliesner, B., Ross, W. R. P. I., Valdes, P. J., Vettoretti, G., Wolk, S. L. W. F., and YU, Y.: Past and future polar amplification of climate change: climate model intercomparisons and ice-core constraints, Clim. Dyn., 513-529, 2006.

Peltier, W.: Global glacial isostasy and the surface of the ice-age Earth: the ICE-5G (VM2) model and GRACE, Ann. Rev. Earth Planet. Sci., 32, 111-149, 2004.

Rougier, J. C.: Probabilistic Inference for Future Climate Using an Ensemble of Simulator Evaluations, Climatic Change, in press, 2006.

Schneider von Deimling, T., Held, H., Ganopolski, A., and Rahmstorf, S.: Climate sensitivity estimated from ensemble simulations of glacial climate, Clim. Dyn., 27, 149-163, 2006.

Stainforth, D. A., Aina, T., Christensen, C., Collins, M., Faull, N., Frame, D. J., Kettleborough, J. A., Knight, S., Martin, A., Murphy, J. M., Piani, C., Sexton, D., Smith, L. A., Spicer, R. A., Thorpe, A. J., and Allen, M. R.: Uncertainty in predictions of the climate response to rising levels of greenhouse gases, Nature, 433, 403-406, 2005.
Tsushima, Y., Emori, S., Ogura, T., Kimoto, M., Webb, M. J., Williams, K. D., Ringer, M. A., Soden, B. J., Li, B., and Andronova, N.: Importance of the mixed-phase cloud distribution in the control climate for assessing the response of clouds to carbon dioxide increase: a multi-model study, Clim. Dyn., 27, 113-126, 2006.

Yokohata, T., Emori, S., Nozawa, T., Tsushima, Y., Ogura, T., and Kimoto, M.: Climate response to volcanic forcing: Validation of climate sensitivity of a coupled atmosphere-ocean general circulation model, Geophys. Res. Lett., 32, L19703, doi:10.1029/2005GL023673, 2005. 